\title{
Abordagem e reflexões para o cuidado do cliente com erisipela
}

\section{Approach and reflections for customer care with erysipelas}

\author{
Ronny Anderson de Oliveira Cruz \\ Graduado em Enfermagem Pela Universidade Federal da Paraíba (UFPB). Especialista em Ativação de Processos de \\ Mudança na Formação de Profissionais de Saúde (FIOCRUZ). Membro do GEPEFE - UFPB. E-mail: \\ ronnyufpb@gmail.com \\ Éder Gonçalves Miranda \\ Acadêmico de Enfermagem da Faculdade Maurício de Nassau - Unidade João Pessoa (PB). E-mail: \\ ederchacal@hotmail.com \\ Edijane da Costa Santos \\ Acadêmica de Enfermagem da Faculdade Maurício de Nassau - Unidade João Pessoa (PB). Membro do GEPEFE - \\ UFPB. E-mail: jane.costa@gmail.com \\ Maria da Guia Martins da Silva Ferreira \\ Acadêmica de Enfermagem da Faculdade Maurício de Nassau - Unidade João Pessoa (PB). Membro do GEPEFE - \\ UFPB. E-mail: maria.silva_@hotmail.com \\ Rogério Alves Santana \\ Acadêmico de Enfermagem da Faculdade Maurício de Nassau - Unidade João Pessoa (PB). E-mail: \\ rogerenf2012@gmail.com
}

\begin{abstract}
RESUMO: Este artigo tem como objetivo realizar uma análise sobre os cuidados específicos prestados a clientes com erisipela a fim de contribuir com o consenso no tocante a melhorias na qualidade da assistência e no alinhamento de condutas. Apresenta abordagem reflexiva, fundamentada a partir de revisão integrativa nas bases de dados Scielo, Lilacs, Bdenf e Medline, por meio dos descritores: Cuidados de enfermagem; Erisipela; Terapêutica; Ferimentos e lesões, nos meses de novembro a dezembro de 2015. A erisipela é uma infecção cutânea aguda de etiologia essencialmente estreptocócica, com episódios de recidiva frequentes. Antes da descoberta da penicilina, verificava-se geralmente uma evolução espontânea para a cura em um período de 01 a 03 semanas, mas associavam-se importantes comprometimentos gerando sequelas e significativa taxa de mortalidade. Após análise dos estudos, emergiram três categorias: Aspectos fisiopatológicos da erisipela, Abordagem interdisciplinar no tratamento da erisipela e A importância do enfermeiro no cuidado ao cliente com erisipela. Através da Sistematização da Assistência de Enfermagem é possível promover um cuidado integral e holístico os diagnósticos e intervenções de enfermagem são de fundamental importância na recuperação e reabilitação melhorando, assim, a qualidade de vida. $\mathrm{O}$ estudo apesar da especificidade dos critérios de inclusão e exclusão possibilitou observar que apesar da alta incidência há atualmente poucos estudos sobre os cuidados ao cliente com erisipela.
\end{abstract}

Palavras chave: Cuidados de Enfermagem; Ferimentos e lesões; Terapêutica.

\begin{abstract}
This article aims to conduct an analysis of the specific care of customers with erysipelas in order to contribute to the consensus with respect to improvements in quality of care and alignment conduits. It features reflective approach, based from integrative review in Scielo databases Lilacs, Medline BDEnf and through the descriptors: nursing care; Erysipelas; Therapy; Wounds and injuries into the months from November to December 2015. The erysipelas is an acute skin infection essentially streptococcal etiology, with frequent episodes of relapse. Before the discovery of penicillin, usually checking It is a spontaneous evolution towards healing in a period 01-03 weeks, but major commitments were associated sequelae and causing significant mortality rate. After analyzing the studies, three categories emerged: pathophysiological aspects of erysipelas, interdisciplinary approach in the treatment of erysipelas and the importance of nurses in the care the customer with erysipelas. Through the systematization of nursing care is possible to promote a comprehensive and holistic care diagnostics and nursing interventions are of fundamental importance in the recovery and rehabilitation, thus improving the quality of life. The study despite the specificity of the inclusion and exclusion criteria made it possible to note that despite the high incidence currently there are few studies on the care the customer with erysipelas.
\end{abstract}

Keywords: Nursing Care; Wounds and injuries; Therapy.

Recebido em 17/12/2015

Aprovado em: 10/02/2016

REBES - ISSN 2358-2391 - (Pombal - PB, Brasil), v. 6, n.1, p. 22-26, Jan-Mar, 2016 


\section{INTRODUÇÃO}

O número de pessoas com lesões de pele vem crescendo bastante em decorrência de vários fatores como o aumento da expectativa de vida acompanhados de hábitos de vida não saudáveis, onde podemos citar o sedentarismo, a má alimentação, tabagismo e a ingestão de bebidas alcoólicas, que consequentemente podem levar a complicações como a hipertensão arterial, a diabetes mellitus e a doenças vasculares, que são fatores agravantes para o surgimento de feridas. As feridas complexas apresentam-se como lesões de difícil resolução, onde a viabilidade dos tecidos se encontra comprometida com isquemia e/ou necrose local, infecções agressivas e associação com doenças sistêmicas que causam prejuízo para os processos normais de cicatrização (COLTRO et al., 2010).

De acordo com a etiologia são classificadas em ferida traumática (incluindo as queimaduras), ferida cirúrgica complicada, ferida necrotizante, úlcera por pressão, úlcera venosa ou arterial, ferida diabética, ferida por vasculite e ferida pós-radiação (FERREIRA et al., 2006).

A erisipela é uma infecção cutânea aguda de etiologia essencialmente estreptocócica, com episódios de recidiva frequentes. É uma celulite superficial com intenso comprometimento do plexo linfático subjacente e se caracteriza por placas eritematosas acompanhadas de dor e edema, onde essas lesões expandem-se perifericamente, tornando-se quentes e com limite demarcado (SILVA et al., 2013).

Ocorre em qualquer faixa etária, e o pico se dá entre 60 e 80 anos. Os locais mais acometidos são os membros inferiores, seguidos da face e membros superiores. É mais frequente em pacientes do sexo feminino, sendo observada na maioria dos casos uma porta de entrada bem definida, como úlceras, traumas, micoses superficiais, picadas de inseto e feridas mal tratadas. As complicações mais frequentes são erisipela bolhosa necrotizante, abscesso, tromboflebite superficial e profunda (ALCANTARA; ALCANTARA, 2009).

O tratamento de feridas necessita cada vez mais de uma intervenção avançada, centrada numa abordagem holística o que norteia os enfermeiros a buscarem fundamentar sua prática baseando-se em evidências, além de uma gestão clínica integrada da ferida e um trabalho multiprofissional. Além disso, face aos produtos disponíveis no mercado atualmente, cabe ao profissional enfermeiro realizar de forma assertiva a escolha de produtos com o intuito de otimizar o processo de cuidar em enfermagem, associando contextos da tecnologia fundamental para seu desenvolvimento científico e social (CAMACHO et al., 2015).

$\mathrm{O}$ processo de trabalho da enfermagem envolve inúmeros elementos em um único processo, por meio do uso de diferentes tecnologias. As inovações tecnológicas favorecem o aprimoramento do cuidado, ressaltando que o cuidado utiliza a tecnologia e não o contrário (CARDOSO; SILVA, 2010).

A Sistematização da Assistência de Enfermagem (SAE) que organiza e executa o Processo de Enfermagem é o caminho onde os enfermeiros procuram formas de envolver e educar suas equipes no intuito de assegurar uma prática assistencial que promova um cuidado de enfermagem individual e integral, fundamentado no conhecimento científico (RAMALHO NETO; FONTES; NÓBREGA, 2013).

Antes da descoberta da penicilina, verificava-se geralmente uma evolução espontânea para a cura em um período de 01 a 03 semanas, mas associavam-se importantes comprometimentos gerando sequelas $\mathrm{e}$ significativa taxa de mortalidade. Com o advento da antibioticoterapia, registrou-se o quase desaparecimento de casos fatais, orientando-se atualmente todos os esforços para o controle efetivo dos fatores de risco, responsáveis pela elevada taxa de recidiva, notável morbidade e considerável dispêndio de recursos econômicos associados a esta infecção (SILVA et al., 2013).

A erisipela é uma patologia frequente na prática clínica, com uma incidência estimada de 10 a 100 casos por 100.000 habitantes/ano. Algumas publicações sugerem o aumento da incidência nas últimas décadas (ALCANTARA; ALCANTARA, 2009).

Com esse ínterim, este estudo baliza-se na seguinte questão norteadora: quais os achados mais relevantes em relação aos cuidados com o cliente com erisipela nos últimos anos?

O propósito deste estudo é apresentar uma análise reflexiva sobre os cuidados específicos prestados a estes clientes a fim de contribuir com o consenso no tocante a melhorias na qualidade da assistência e no alinhamento de condutas.

\section{METODOLOGIA}

Estudo descritivo, tipo análise reflexiva a partir de levantamento bibliográfico, desenvolvido por meio da consulta em bases de dados eletrônicas. Foram realizadas consultas a periódicos na base de dados Literatura LatinoAmericana e do Caribe em Ciências da Saúde (LILACS), Base de Dados de Enfermagem (BDENF), Medical Literature Analysis and Retrieval System Online (MEDLINE) e Scientific Electronic Library Online (SCIELO), por meio dos descritores: Cuidados de enfermagem; Erisipela; Terapêutica; Ferimentos e lesões, nos meses de novembro a dezembro de 2015. Os critérios de inclusão foram: trabalhos completos, disponíveis online, nas línguas portuguesa, publicados nos últimos 15 anos e que contemplassem informações acerca da questão norteadora. Para análise utilizou-se a técnica de análise textual discursiva dos artigos que atenderam aos critérios de inclusão. Essa técnica consiste em descobrir os núcleos de sentido que compõem uma comunicação, cuja presença ou frequência acrescentem perspectivas significativas ao objeto de estudo em questão. A noção da temática está associada a uma afirmação que diz respeito a um determinado assunto, podendo ser apresentada por uma palavra, frase ou ideia (MORAES; GALIAZZI, 2011). Após análise dos estudos, emergiram três categorias: Aspectos fisiopatológicos da erisipela, Abordagem interdisciplinar no tratamento da erisipela e A importância do enfermeiro no cuidado ao cliente com erisipela. 


\section{REVISÃO BIBLIOGRÁFICA}

\section{Aspectos fisiopatológicos da erisipela}

Trata-se de uma infecção cutânea causada geralmente pelo estreptococos $b$ - hemolítico, do grupo A, menos freqüente do grupo $\mathrm{C}$ ou $\mathrm{G}$ e, mais raramente, por Staphylococcus aureus. A erisipela por estreptococos bhemolítico, do grupo A é a causa mais comum de infecção grave dos tecidos moles, que penetram através de um pequeno ferimento na pele ou na mucosa, disseminam-se pelos vasos linfáticos e podem atingir o tecido subcutâneo (SOUZA, 2003).

A condição é caracterizada por hiperemia, edema, dor e aumento da temperatura no local da lesão, acompanhado por febre, calafrios, mal-estar e muitas vezes náuseas ou vômitos. A lesão é geralmente única, elevada, média de 10 a $15 \mathrm{~cm}$ em seu maior eixo, com uma fronteira clara, que avança com a progressão da doença. As bolhas podem desenvolver, geralmente flácida, com um conteúdo translúcido sendo o diagnóstico basicamente alcançado através de exame clínico, leucocitose leve com desvio a esquerda e eritrócitos com taxa de sedimentação ligeiramente aumentada. Histologicamente é caracterizada por edema da derme e dilatação linfática e o diagnóstico diferencial se dá com a fasciíte necrosante, dermatite de contato e trombose venosa profunda (EMPINOTTI et al., 2012).

O exsudato de proteínas, a fibrina, os elementos figurados, bem como as lesões endoteliais os quais conduzem a trombose troncular linfática, acabam levando ao edema e linfedema. Cerca de $85 \%$ dos linfedemas devem-se à linfangite de repetição e, por isso, deve-se sempre considerar esse diagnóstico quando se está diante de quadro dermatológico associado a comprometimento linfático (linfedemas, linfangites). O linfedema crônico por erisipelas pode levar à deformidade caracterizando o quadro de elefantíase mostra (FERNANDES; FLEURY JÚNIOR, 2011).

As complicações ocorrem em percentual que varia de 8 a $30 \%$ dos casos, podendo manifestar-se como áreas de necrose, abscessos, gangrena, fasciíte necrotizante, tromboflebite, glomerulonefrite aguda, septicemia, artrite séptica, endocardite e até morte. A evolução é favorável em 80 a $90 \%$ dos casos, e a mortalidade pode variar de 0,5 a $20 \%$ dos casos, dependendo do antibiótico usado e das comorbidades associadas. Recidivas em até seis meses podem estar presentes em 12\% dos casos (OKAJIMA; FREITAS; ZAITZ, 2004).

\section{Abordagem interdisciplinar no tratamento da erisipela}

A reparação de um tecido exige um ambiente propicio para a formação de colágeno, angiogênese e epitelização da ferida. Fatores sistêmicos e locais podem interferir nesse processo como: idade, sexo, doenças oncológicas, insuficiência renal e vascular, diabetes mellitus, estado nutricional, baixa resistência, técnica de limpeza, infecção e cobertura utilizada (ALCANTARA; ALCANTARA, 2010).

A condição nutricional é um fator decisivo na evolução do processo cicatricial devido à especificidade de elementos, como as proteínas que favorecem a resposta inflamatória e a síntese de colágeno com a remodelação da ferida, os carboidratos que fornecem energia aos leucócitos e fibroblastos, as gorduras que reservam energia, as vitaminas $\mathrm{A}$ e $\mathrm{C}$ que promovem a linfócitose e a síntese de colágeno e epitelização. A vitamina $\mathrm{K}$ que atua no processo da coagulação, o complexo B que favorece a ligação do colágeno, o zinco que auxilia na proliferação celular epitelização além de aumentar a resistência do colágeno, e o manganês, cobre e magnésio que contribuem na síntese do colágeno (POLETTI; CALIRI, 2005).

Nesse contexto, é cabível salientar que a assistência ao cliente que apresenta uma ferida seja ela uma erisipela ou não, deve ser realizada por uma equipe multiprofissional, a qual envolve a participação de outros profissionais além do enfermeiro, como o nutricionista, o fisioterapeuta, o psicólogo, o farmacêutico clínico, assistente social e o médico. Faz-se indispensável à presença de todos neste processo, tendo em vista que cada um assume um papel de relevância, possibilitando, através de métodos terapêuticos aplicados ao paciente, promover sua cicatrização e bem-estar. (MORAIS; OLIVEIRA; SOARES, 2008).

Nas relações profissionais existentes entre médicos e as demais categorias profissionais, é evidente em muitos relatos a questão da autoridade médica no campo da saúde. Tratando-se de um fator histórico, passa a ser de extrema relevância, sobretudo em áreas que se apresentam predominantemente composta por mulheres, em que o ideal feminino subordina-se a representação social do seu papel tradicional de mãe, reforçando as tradições e o perfil da maternidade. Contudo, entendemos que situações como esta não se limitam apenas a questão de gênero, mas envolve uma hierarquia complexa, em que se interpõem valores éticos entre os profissionais que exercem atividades distintas.

\section{A importância do enfermeiro no cuidado ao cliente com erisipela}

A prática de cuidados a pessoas com feridas é uma especialidade dentro da enfermagem, reconhecida pela Sociedade Brasileira de Enfermagem em Dermatologia (SOBEND) e Associação Brasileira de Estomaterapia (SOBEST) e, ao mesmo tempo é um desafio que requer conhecimento específico, habilidade e abordagem holística. O cuidado de enfermagem em feridas requer atenção especial por parte dos profissionais da saúde, destacando-se o papel do enfermeiro, que busca novos conhecimentos para fundamentar sua prática (BRITO et al., 2013).

Os enfermeiros devem planejar, executar e avaliar a assistência de enfermagem ao cliente, tomando por base os aspectos filosóficos, técnicos e científicos, utilizando a metodologia da assistência. Dessa forma, os enfermeiros atuam tanto na prevenção como na avaliação, fortalecendo as práticas preventivas em saúde com vistas ao cuidado individualizado. Evidencia-se que as ações do enfermeiro atualmente envolvem o princípio da integralidade e sendo assim preza o olhar holístico do cuidado. Vale ainda ressaltar que os profissionais de saúde, em particular os enfermeiros, estão envolvidos no 
gerenciamento de materiais e insumos das unidades de saúde, e consequentemente, nos seus custos. Portanto, é importante conhecer os gastos relacionados ao tratamento de feridas (JESUS; BRANDÃO; SILVA, 2015).

Entretanto, a melhor forma de se evitar os altos custos destinados ao tratamento das lesões reside no processo de prevenção destas. Para tanto, a enfermagem só poderá atuar adequadamente na prevenção das feridas se apresentar condições de detectar os fatores de risco predisponentes nos pacientes e se houver o reconhecimento dos prejuízos ao paciente e também à instituição de saúde (BRITO et al., 2013).

E para intervir no processo de cicatrização das feridas, atualmente existe uma variedade de insumos disponíveis no mercado, que pode ser indicado de acordo com o tipo de feridas. Dentre os mais utilizados estão os filmes de poliuretano, hidrocolóides, hidrogeis, papaína, carvão ativado, alginatos, enzimas proteolíticas, ácidos graxos essenciais e derivados de prata (CAMPOS; MORE; ARRUDA, 2007).

Em relação à escolha do curativo vários autores afirmam que a seleção da cobertura ideal deve ser baseada nas características da ferida como: o leito, quantidade e aspecto do exsudato, presença ou não de infecção, o resultado encontrado neste estudo demonstra que ao utilizar um mesmo tipo de cobertura, nas diferentes fases da ferida, o processo de evolução foi efetivo. É importante salientar que o curativo deve proporcionar umidade à ferida, para facilitar o processo de cicatrização, absorver o excesso de exsudato, ser fácil de trocar, impermeável a microorganismos, e reduzir a dor (BAHIA, 2010).

A penicilina cristalina (PC) é a droga de escolha para o tratamento de clientes que necessitam de hospitalização. A utilização de antibióticos de amplo espectro representa maior custo quando comparada ao uso da PC. Os pacientes que fizeram uso de PC apresentam menor tempo de internação, bem como tendência a uma menor incidência de complicações. $O$ uso de anticoagulantes é indicado pela maioria dos autores na presença ou suspeita de trombose venosa profunda ou tromboflebite, mas não em sua profilaxia, entretanto, autores relatam que clientes que fizeram uso de anticoagulante apresentaram menor incidência de complicações quando comparados aos que não utilizaram essa terapia (OKAJIMA; FREITAS; ZAITZ, 2004).

Os diagnósticos de enfermagem comumente encontrados são: dor aguda, hipertermia, integridade da pele prejudicada, integridade tissular prejudicada, ansiedade, excesso de volume de líquidos, baixa autoestima, risco de infecção, mobilidade física prejudicada, intolerância a atividade (NANDA, 2013).

\section{CONSIDERAÇÕES FINAIS}

O cuidado no tratamento de feridas tem exigido cada vez mais dos profissionais envolvidos a perspectiva de uma atenção interdisciplinar. Com novas tecnologias e produtos é possível alcançar melhores resultados e intervir com maior eficiência e eficácia principalmente nas estratégias de prevenção, avaliação e tratamento.

A erisipela é um problema grave e muito comum nas unidades da Atenção Primária em Saúde, bem como nos demais níveis de atenção com potencial incapacitante pela possibilidade de comprometer outros órgãos, podendo evoluir para a sepse e até ao óbito se não tratada.

Através da Sistematização da Assistência de Enfermagem é possível promover um cuidado integral e holístico permitindo que o cliente sinta-se acolhido e corresponsável pelo seu tratamento com vistas à promoção do autocuidado. Os diagnósticos e intervenções de enfermagem são de fundamental importância na recuperação e reabilitação melhorando, assim, a qualidade de vida.

O estudo apesar da especificidade dos critérios de inclusão e exclusão possibilitou observar que apesar da alta incidência há atualmente poucos estudos sobre o cliente com a erisipela. Portanto é imprescindível estudo e conhecimento de novas opções de coberturas disponíveis no mercado, compreensão das condições vivenciadas pelos clientes, ampliação da oferta e do acesso profissional a recursos materiais adequados e treinamentos específicos para que possam desenvolver um trabalho interdisciplinar com as condições necessárias para o estabelecimento de condutas terapêuticas eficazes neste processo.

\section{REFERÊNCIAS BIBLIOGRÁFICAS}

ALCANTARA, C.; ALCANTARA, V.C.S. Cicatrização de lesões causadas por erisipela em um paciente diabético. Com. Ciências Saúde. Vol. 20, n. 2, p. 173-184, 2009. Disponível

em: http://www.escs.edu.br/pesquisa/revista/2010Vol21_2art1 0Cicatrizacaoaodelesoes.pdf. Acesso em: 30 nov. 2015.

BAHIA, Secretaria da Administração. Protocolo Clínico de Feridas/ Normas e Diretrizes para Prevenção e Tratamento de Feridas. Salvador, 2010.

BRITO, K.K.G.; SOUSA, M.J.; SOUSA, A.T.O.; MENESES, L.B.A.; OLIVERIA, S.H.S.; SOARES, M.J.G.O. Feridas crônicas: abordagem da enfermagem na produção científica da pós-graduação. Rev enferm UFPE on line. Vol. 7, n. 2, p. 414-21, 2013. Disponível em: http://www.revista.ufpe.br/revistaenfermagem/index.php/r evista/article/download/3432/5310. Acesso em: $12 \mathrm{dez}$. 2015.

CAMACHO, A.C.L.F.; SANTOS, R.C.; JOAQUIM, F.L.; LOUREDO, D.S.; MORAIS, I.M.; SILVA, E.A. Estudo comparativo sobre a capacidade funcional de pacientes adultos e idosos com úlceras venosas. J. res.: fundam. care. Online. Vol. 7, n. 1, p. 1954-66, 2015. Disponível em:

http://www.seer.unirio.br/index.php/cuidadofundamental/ article/viewFile/3505/pdf_1435. Acesso 30 nov. 2015.

CAMPOS, A.A.G.; MORE, L.F.; ARRUDA, S.S. Protocolo de cuidados de feridas. Florianópolis: IOESC, 2007.

CARDOSO, G.B.; SILVA A.L.A. O processo de trabalho na enfermagem: articulação das tecnologias do cuidado. Revista de Enfermagem UERJ. Vol. 18, n. 3, p. 451- 5, 2010. Disponível em: http://www.facenf.uerj.br/v18n3/v18n3a20.pdf. Acesso 02 dez. 2015. 
COLTRO, P.S.; FERREIRA, M.C.; BATISTA, B.P.S.N.B.; NAKAMOTO, H.A.; MILCHESKI, D.A.; JÚNIOR, P.T. Tratamento cirúrgico das feridas complexas: experiência da cirurgia plástica no Hospital das Clínicas da FMUSP. Rev Medicina. Vol. 89n. 3/4, p. 153-7, 2010. Disponível em: http://www.revistas.usp.br/revistadc/article/view/46290.

Acesso em: 20 nov. 2015.

EMPINOTTTI, J.C.; UYEDA, H.; RUARO, R.T.; GALHARDO, A.P.; BONATTO, D.C. Piodermites. An Bras Dermatol. Vol. 87, n. 2, p. 277-84, 2012. Disponível em: http://www.scielo.br/scielo.php?pid=S036505962012000200013\&script=sci_abstract\&tlng=pt.

Acesso em: $11 \mathrm{dez} .2015$.

FERNANDES, L.B.; FLEURY JUNIOR, L.F.F. Dermatologia comparativa: similaridade entre elefantíase nostra verrucosa e coral. An. Bras. Dermatol. Vol.86, n.4, p. 825-826, 2011. Disponível em: http://www.scielo.br/scielo.php?script=sci_arttext\&pid=S 0365-05962011000400039. Acesso em: 04 dez. 2015.

FERREIRA, M.C.; TUMA, Jr.P.; CARVALHO, V.F.; KAMAMOTOT, F. Complex wounds. Clinics. Vol. 61, n. 6, p. 571-8, 2006. Disponível em: http://www.scielo.br/scielo.php?pid=S1807-

59322006000600014\&script=sci_arttext. Acesso em 20 nov.2015.

JESUS, P.B.R.; BRANDÃO, E.S.; SILVA， C.R.L. Cuidados de enfermagem aos clientes com úlceras venosas uma revisão integrativa da literatura. J. res.: fundam. care. online. Vol. 7, n. 2, p. 2639-48, 2015 Disponível em: http://www.seer.unirio.br/index.php/cuidadofundamental/ article/viewFile/2176/pdf_1561. Acesso em: 12 dez. 2015.

MORAES, R; GALIAZZI, M.C. Análise textual discursiva. 2nd ed. rev. Ijuí: Editora Unijuí; 2011.

MORAIS, G.F.C.; OLIVEIRA, S.H.S.; SOARES, M.J.G.O. Avaliação de feridas pelos enfermeiros de instituições hospitalares da rede pública. Texto Contexto Enferm. Vol. 17, n. 1, p. 98-105, 2008. Disponível em: http://www.scielo.br/scielo.php?pid=S0104-

07072008000100011\&script=sci_abstract\&tlng=pt.

Acesso em: 14 dez. 2015.

NANDA Internacional. Diagnósticos de enfermagem da NANDA: definições e classificação 2012-2014. Porto Alegre: Artmed; 2013

OKAJIMA, R.M.O.; FREITAS, T.H.P.; ZAITZ, C. Estudo clínico de 35 pacientes com diagnóstico de erisipela internados no Hospital Central da Irmandade da Santa Casa de Misericórdia de São Paulo. An. Bras. Dermatol. vol. 79, n.3, p. 295-303, 2004. Disponível em: http://www.scielo.br/pdf/abd/v79n3/v79n3a05.pdf. Acesso em: 11 dez. 2015.

POLETTI, N.A.A.; CALIRI, M.H.L. Atenção a Pacientes com Feridas: evidências para a prática. São José do Rio Preto; 2005.
RAMALHO NETO, J.M.; FONTES, W.D.; NÓBREGA, M.M.L. Instrumento de coleta de dados de enfermagem em Unidade de Terapia Intensiva Geral. Rev Bras Enferm. Vol. 66, n. 4, p. 535-42, 2013. Disponível em: http://www.scielo.br/scielo.php?pid=S0034-

$71672013000400011 \&$ script=sci_arttext. Acesso em: 03 dez. 2015 .

SILVA，P.L.N.; ABREU, G.G.D.; FONSECA, J.R.; SOUTO, S.G.T.; GONÇALVES, R.P.F. Diagnóstico e intervenções de enfermagem em paciente com erisipela: estudo de caso em um hospital de ensino. Revista Eletrônica Gestão \& Saúde. Vol. 4, n. 4, p. 1512-26, 2013. Disponível em: http://gestaoesaude.bce.unb.br/index.php/gestaoesaude/art icle/view/591. Acesso em: 23 nov. 2015.

SOUZA, C.S. Infecções de tecidos moles - Erisipela. Celulite. Síndromes infecciosas mediadas por toxinas. Medicina (Ribeirão Preto). Vol. 1, n. 36, p. 351-356, 2003. Disponível em: http://www.revistas.usp.br/rmrp/article/view/733. Acesso em 04 dez. 2015. 